\title{
Variation in Emission and Energy Recovery Concerning Incident Angle in a Scheme Recovering High Energy Ions by Secondary Electrons*)
}

\author{
Takayuki WADA, Shota KONNO, Satoshi NAKAMOTO, Hiromasa TAKENO, \\ Yuichi FURUYAMA ${ }^{1)}$ and Akira TANIIKE ${ }^{1)}$ \\ Electrical and Electronic Engineering, Kobe University, Kobe 657-8501, Japan \\ ${ }^{1)}$ Graduate School of Maritime Sciences, Kobe University, Kobe 658-0022, Japan
}

(Received 29 November 2015 / Accepted 2 February 2016)

\begin{abstract}
As an energy recovery device for fast protons produced in $\mathrm{D}-{ }^{3} \mathrm{He}$ nuclear fusion, secondary electron (SE) direct energy converter (SEDEC) was proposed in addition to traveling wave direct energy converter (TWDEC). Some protons passing through a TWDEC come into an SEDEC, where protons penetrate to a number of foil electrodes and emitted SEs are recovered. Following to a development of SE orbit control by magnetic field, dependence on incident angle of protons was examined to optimize structure of SEDEC. Based on a theoretical expectation, experiments were performed by changing incident angle of protons and variation in emission and energy recovery were measured. Both emission and energy recovery increased as the angle increased, and differences with theoretical expectation are discussed.
\end{abstract}

(c) 2016 The Japan Society of Plasma Science and Nuclear Fusion Research

Keywords: advanced fusion, direct energy conversion, secondary electron, SEDEC

DOI: $10.1585 /$ pfr.11.2405029

\section{Introduction}

D- ${ }^{3} \mathrm{He}$ nuclear fusion has been expected as an energy source for next generation because neutrons are not produced in this reaction. Furthermore, most of the energy is released as the kinetic energy of charged particles, thus direct energy conversion (DEC) can be applied. An energy recovery device for produced protons by using traveling wave direct energy converter (TWDEC) was proposed [1]. However, it was revealed some protons were not decelerated and efficiency of the device was limited. To recover the protons passing through TWDEC, an introduction of an additional new device was proposed to be settled in the downstream of the TWDEC, which was called secondary electron (SE) direct energy converter (SEDEC) [2]. In an SEDEC, a number of foil electrodes are aligned in the direction of the proton beam, and the protons penetrate them when SEs are emitted. The SEs are caught by appropriately biased collectors and energy of the SEs is recovered, which means energy of the protons is indirectly recovered by collecting SEs. The device is an additional one as its independent efficiency is not high.

SEDEC was developed by a series of experiments. In order to control orbits of SEs, magnetic field was introduced by permanent magnets (PMs) [3]. This scheme was improved that PMs were settled only on one side and by taking an appropriate distance to the collector plate to re-

author'se-mail: takeno@eedept.kobe-u.ac.jp

*) This article is based on the presentation at the 25th International Toki Conference (ITC25). duce reflection in front of the PMs as shown in Fig. 1. In this structure, the amount of the recovered energy exceeded that without PMs [4].

For the optimization of the structure of SEDEC, there still remain various conditions such as foil thickness, interval of foils, and so on. In this paper, we treat angle of the incident beam. The theoretical dependence of the amount of SE is as follows [5].

$$
\Gamma(\theta)=\Gamma(0) \exp \left[A_{\mathrm{s} 0}(1-\cos \theta)\right],
$$

where $\Gamma(\theta)$ is the amount of SE at incident angle of $\theta$ and 1.45 is taken as $A_{\mathrm{s} 0}$ in Ref. 5. According to Eq. (1), the amount of SE increases as $\theta$ increases. Increase of SE directly affects energy recovery, so the incident angle is one of the important parameters.

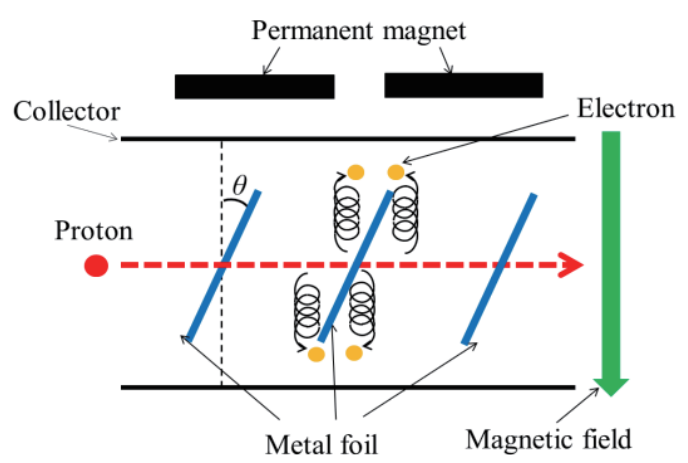

Fig. 1 Improved structure of SEDEC with PMs. 


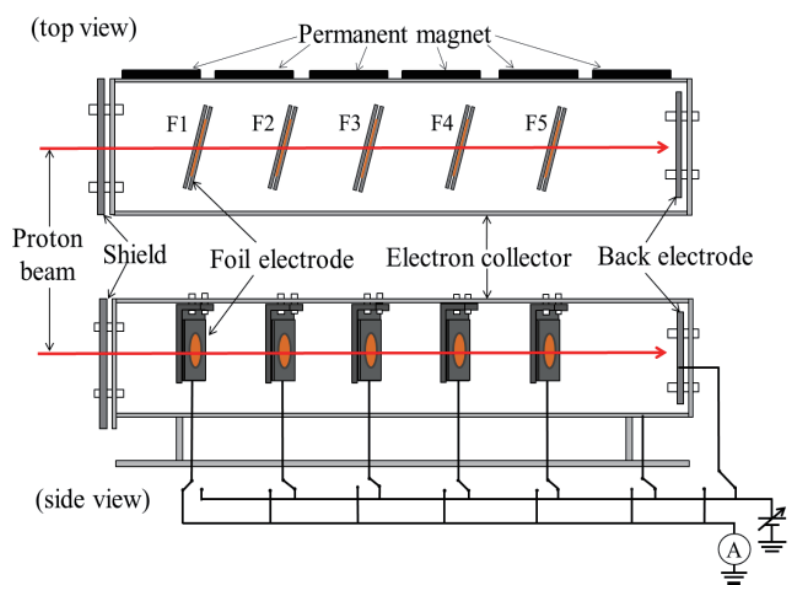

Fig. 2 Fundamental structure of an experimental device of SEDEC.

This paper presents experiments to examine variation in emission and energy recovery concerning incident angle. The effectiveness of magnetic field application is also confirmed on a deep incident angle.

In Sec. 2, experimental equipments are explained. The results of the experiments with discussions are shown in Sec. 3. The contents of the paper are summarized in Sec. 4 .

\section{Experimental Equipments}

In the experiment, we used a tandem electrostatic accelerator (NEC 5SDH-2) as a high energy proton source. The energy of the proton beam used was $2.3 \mathrm{MeV}$ and the beam was masked by an aperture with $5 \mathrm{~mm}$ diameter.

The fundamental structure of an experimental device of SEDEC is shown in Fig. 2. Five foil electrodes and a back electrode were settled in a container. The foil electrodes were made of aluminum. All plates of the container were made of stainless steel and connected electrically. The whole of the container was used as an electron collector. The container was settled under the pressure of 3 - 9 $\times 10^{-4} \mathrm{~Pa}$.

The measurement circuit is also shown in Fig. 2, by which a current-voltage (I-V) characteristic for each electrode was measured. It should be noted that the meaning of I-V characteristic is unusual: the voltage was applied to all electrodes except the target (current measuring) electrode. For example in Fig. 2, F2-F5, the back electrode, and the container were biased to obtain the I-V characteristic of F1. When bias voltage was negative, the target electrode was relatively in positive potential, so electrons flew into the target electrode and negative current was observed. The ion source of the accelerator had a low frequency fluctuation and measured current was also fluctuated. The beam current monitored by a Faraday cup located upstream of the SEDEC was fluctuating in the orders of $\mathrm{nA}$ and $10 \mathrm{nA}$. The target current was proportionally normalized by the beam current of $1 \mathrm{nA}$.

The foil electrodes and PM arrangement were

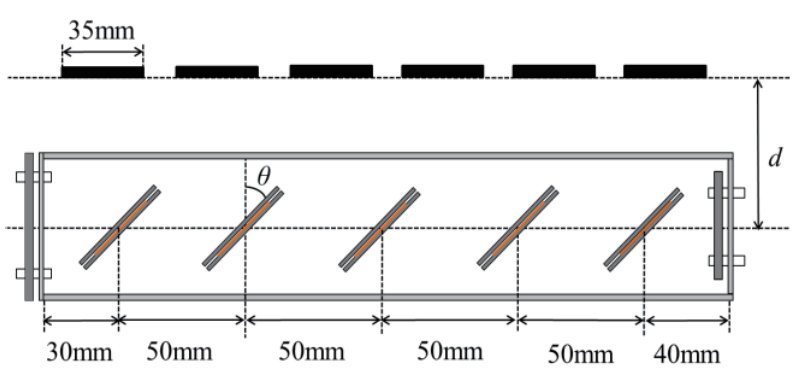

Fig. 3 Arrangement of foil electrodes and PMs.

changed according to experimental conditions. As for the angle of $\theta$ in Fig. 3, which corresponded to the incident angle to the foil electrode, we examined three cases: 15,30 , and 45 degrees. Foil thickness of F2-F5 in Fig. 2 was $5 \mu \mathrm{m}$, and that of F1 in Fig. 2 depended on $\theta: 18 \mu \mathrm{m}$ in the case of 15 and 30 degrees and $12.5 \mu \mathrm{m}$ in the case of 45 degrees. The thickness of F1 was determined by taking proton energy reduction due to penetration into account [2].

As for PMs, there were three patterns. The first one was no arrangement of PMs. The second one was taking $26 \mathrm{~mm}$ to the distance between the beam axis and PMs ( $d$ in Fig. 3), and the third one was $d=146 \mathrm{~mm}$. The shape of one PM was a rectangular parallelepiped, and its width, height, and thickness were 35,20 , and $5 \mathrm{~mm}$, respectively. The magnetization was in the direction of thickness, and the surface magnetic field was $270 \mathrm{mT}$. Six pieces of PMs were aligned in the direction parallel to the beam axis.

\section{Experimental Results and Discus- sion} 3.1 Emission of SE

In Fig. 4, I-V characteristics of foil electrodes are shown. Here, (a), (b), and (c) are for $\theta=15,30$, and 45 degrees, respectively. No PMs were arranged for all cases. According to Fig. 4, currents saturate in the large positive bias voltage region for all characteristics. In this region, the measuring electrode is relatively in deep negative, so all emitted SEs go to other electrodes. The saturating current is considered to correspond to the amount of emitted SEs, and we use the current at $+150 \mathrm{~V}$ for it.

When we compare (a), (b), and (c) of Fig. 4, the amount of emitted SEs increases as angle $\theta$ increases. We also performed measurements for other patterns of PM arrangement. By taking a sum of the amount of emitted SEs for each condition, its dependence on $\theta$ is summarized in Fig. 5. The amount of emitted SEs is normalized by that for the case of $\theta=15$ degrees corresponding to the same pattern of PM arrangement, and the ordinate of the graph takes the normalized value. The curve in the figure shows theoretical value calculated by Eq. (1), which is also normalized by $\Gamma(\theta=15$ degrees $)$. Although 1.45 is taken as $A_{\mathrm{s} 0}$ in Eq. (1) in Ref. 5, this value is based on the assumption, and it is not clear in the parameter range of the present experiment. We assume three kinds of $A_{\mathrm{s} 0}$, and present the- 


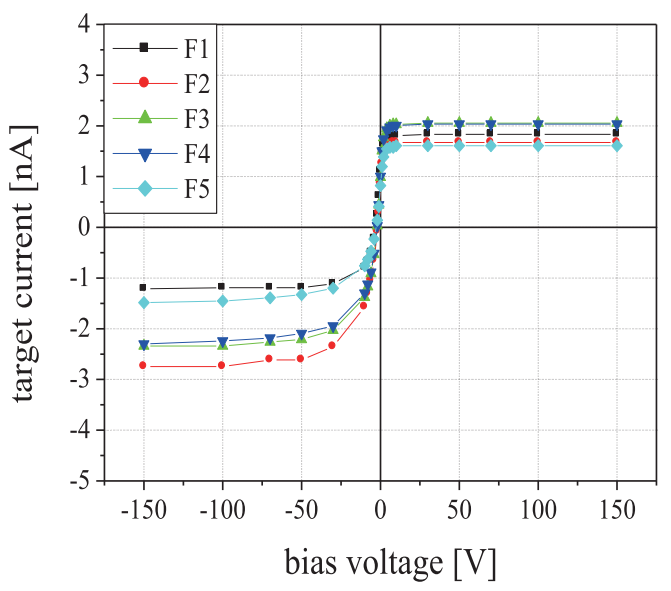

(a) $\theta=15$ degrees

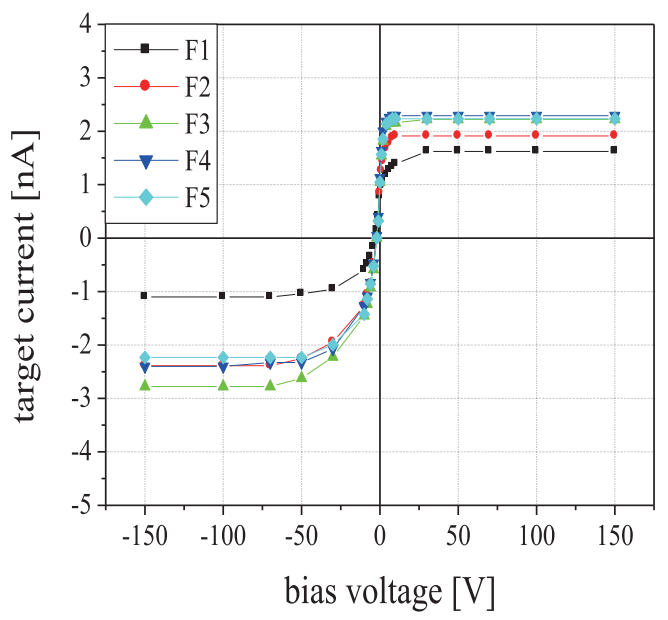

(b) $\theta=30$ degrees

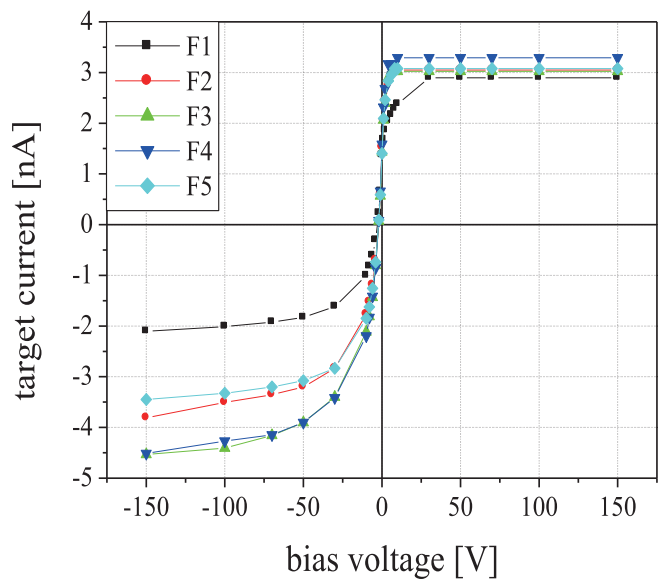

(c) $\theta=45$ degrees

Fig. 4 I-V characteristics of foil electrodes for no PMs arrangement.

oretical curves in Fig. 5.

According to Fig. 5, the amount of emitted SEs increases as $\theta$ increases which qualitatively agrees with the theoretical dependence. Quantitative agreement, however, is not so well. It is relatively less on $\theta=30$ degrees and more on $\theta=45$ degrees, and this is common for all patterns of PM arrangement. As for the reason of this phenomenon, we will discuss later.

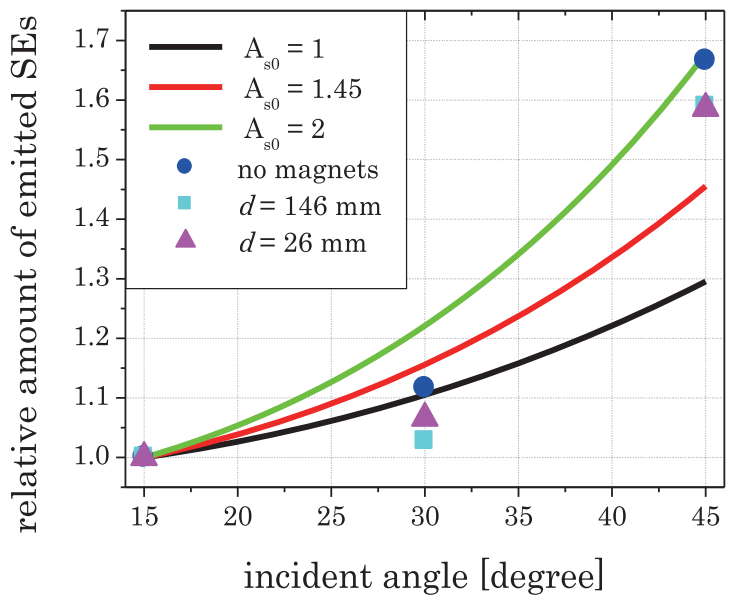

Fig. 5 Amount of emitted SEs versus incident angle.

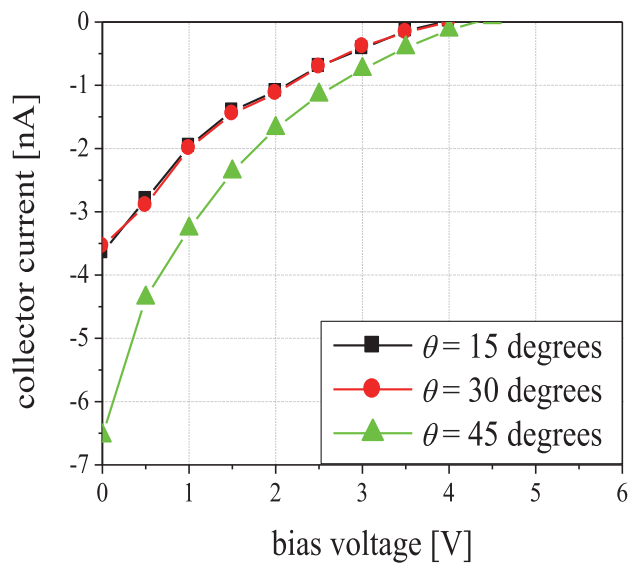

(a) no PMs

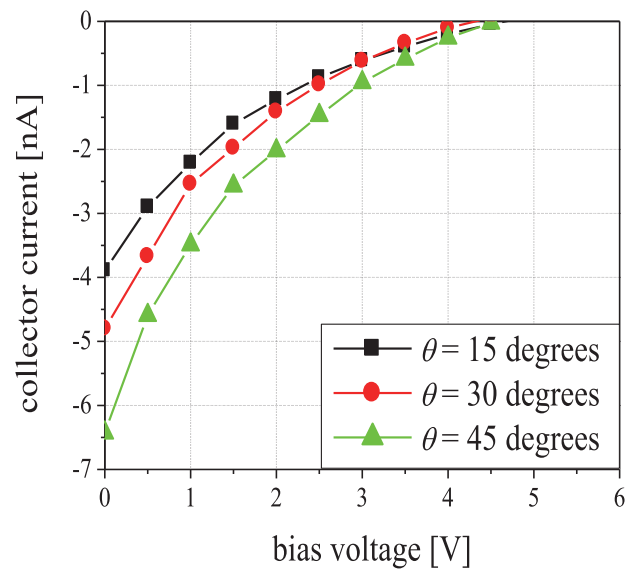

(b) $d=146 \mathrm{~mm}$

Fig. $6 \mathrm{I}-\mathrm{V}$ characteristics of the collector.

\subsection{Collection of SE and discussion}

The I-V characteristics were also measured on the collector. In the result, the characteristic in the fourth quadrant relates energy recovery of SEs. The meaning of the measured current in this region is the amount of SEs arriving at the collector against retarding field (positive bias on the other electrodes than the collector).

In Fig. 6, I-V characteristics of the collector in the 


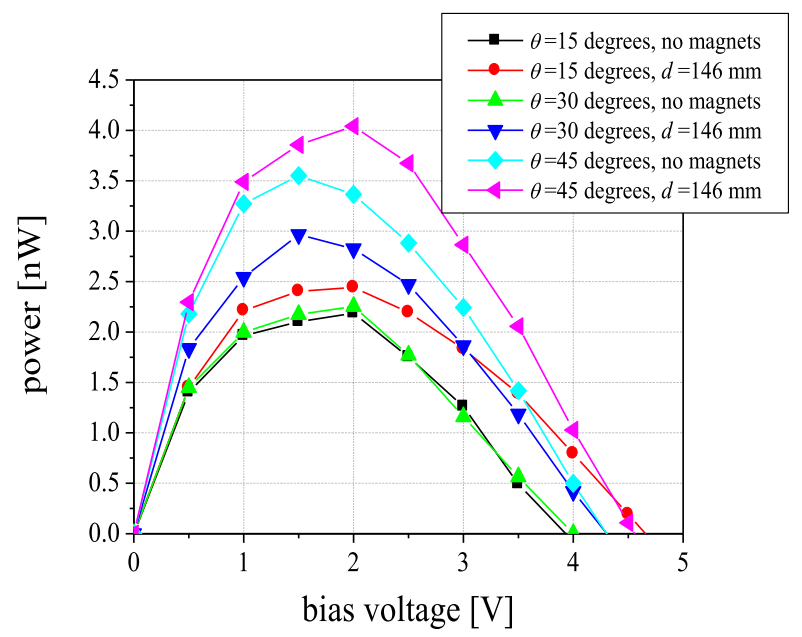

Fig. 7 Power-voltage characteristics.

fourth quadrant in the cases of (a) no PMs and (b) PMs with $d=146 \mathrm{~mm}$ are shown. According to Fig. 6 , the largest negative current is obtained on $\theta=45$ degrees in both conditions. The amount of SEs reaching the collector can be increased by increasing $\theta$.

The amount of recovered power was evaluated by the product of the bias voltage and the absolute value of collector current in the fourth quadrant. In Fig. 7, power-voltage (P-V) characteristics of no PMs and PMs with $d=146 \mathrm{~mm}$ are summarized. It is found that the amount of recovered power in the case of $\theta=45$ degrees is large, and that for PMs with $d=146 \mathrm{~mm}$ is larger than that for no PMs. It is confirmed that increasing incident angle is effective in terms of energy recovery, and an appropriate magnetic field application is also effective even on a deep incident angle.

The practical device works in the optimum condition, thus, we take the maximum recovered power as an optimum power. We evaluate the maximum power in the $\mathrm{P}$ $\mathrm{V}$ characteristic and normalize the values by those for the cases of $\theta=15$ degrees. They are relative optimum power and summarized in Fig. 8 as a function of $\theta$. As the variation of the amount of emitted SEs directly affects recovered power, and thus the relative optimum power, theoretical curves based on Eq. (1) are also indicated in the same manner as that in Fig. 5. The measured results qualitatively agree with the theory, but they have some discrepancy quantitatively.

In the case of $\theta=30$ degrees, relative difference between measured value and theoretical one is not consistent with that in Fig. 5, and this means that not only increment of the amount of emitted SEs, but also some other factors such as difference of electron orbit may affect energy recovery. However, increment of relative optimum power or

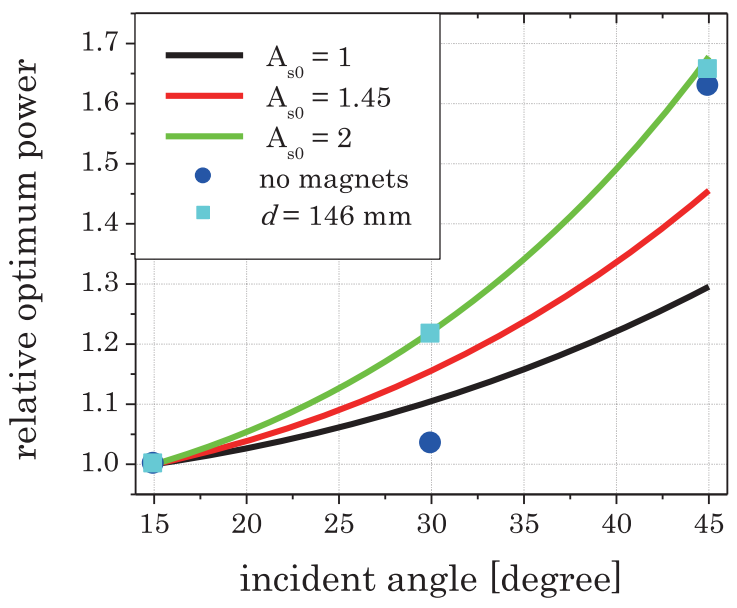

Fig. 8 Relative optimum power versus incident angle.

emitted SEs in the case of $\theta=45$ degrees are much larger than those on $\theta=30$ degrees.

As shown above, increase of incident angle is effective for improvement of energy recovery. The precise dependence on incident angle, however, is rather complex. The referred Eq. (1) represents only for back scattering, but forward scattering should be taken into account. Protons completely penetrate foil electrodes in the present scheme, and the forward scattering was confirmed in Ref. 4. The theory of SE emission should be developed.

\section{Summary}

Toward optimization of the structure of SEDEC, we examined the dependence on the beam incident angle. The amount of emitted SEs and recovered power, and thus the optimum power increased as the incident angle increased. These results roughly agree with the theory of SE emission, however, the detailed experimental information as well as development of the theory are required to optimize the device.

\section{Acknowledgment}

The authors acknowledge valuable discussions with Drs. J. Miyazawa and T. Goto.

[1] H. Momota, LA-11808-C, Los Alamos Natl. Lab., 8 (1990).

[2] D. Akashi et al., Trans. Fusion Sci. Tech. 63, 301 (2013).

[3] Y. Takeshita et al., 9th General Scientific Assembly of the Asia Plasma and Fusion Association in 2013, TP-36 (2013).

[4] S. Hagihara et al., Plasma Fusion Res. 10, 3405025 (2015).

[5] G. Fubiani, H.P.L. de Esch, A. Simonin and R.S. Hemsworth, Phys. Rev. ST Accel. Beams 11, 014202 (2008). 